\title{
Reliability management and computing
}

\author{
Hoang Pham ${ }^{1}$
}

Published online: 19 July 2016

CC Springer Science+Business Media New York 2016

Most of the products that affect our daily lives are becoming more complex. Reliability management-which integrates processes, policies, and reliability predictions from the beginning of the product development life cycle to ensure high levels of product performance and safety-helps companies address the challenges of increasingly complex systems and software and globally widespread processes in today's competitive marketplace.

This special issue on reliability management and computing consists of 13 outstanding papers that address various research challenges in reliability management and computing related areas including optimization, maintenance, network reliability, replacement policies, software reliability and measurements, change-point modeling, renewal processes, statistical inference, fault-tolerant computing, etc.

The first paper, by Chang, presents a new allocation approach that orders weighted averaging tree and soft set methods to allocate the reliability of individual subsystems in order to achieve the reliability objective of the entire system. The paper by Sheu et al. presents a cumulative damage replacement model considering a two-unit system with failure interactions subject to two types of shocks based on nonhomogeneous Poisson processes. The optimal replacement policy that minimizes the expected cost per unit time is discussed. The paper by Niu et al. discusses a new maintenance model of a repairable deteriorating system based on a renewal geometric process using the renewal-reward theorem. The authors also discuss the optimal replacement policies for the proposed geometric-process maintenance model that minimizes the average cost rates.

The paper by Lin, Yeh, and Huang develops a network model and algorithms to evaluate supply-chain reliability for brittle commodity logistics, in which the network is composed of multiple suppliers and carriers. An application of a flat glass logistics network is also discussed to illustrate the proposed algorithm. The paper by Minamino, Inoue, and Yamada discusses the optimal software release policies of software reliability growth models addressing the change-points based on a nonhomogeneous Poisson process that minimizes the expected total software cost.

$凶$ Hoang Pham hoang84pham@gmail.com

1 Rutgers University, Piscataway, NJ 08854, USA 
The paper by Zhao and Nakagawa focuses on comparisons of over-time, over-level replacement costs with random working cycles. The paper by Park, Jung, and Park proposes a warranty cost model with consideration of both repair service and replacement service upon the system failure to determine the optimal warranty period that minimizes the expected cost rate during the warranty cycle. The paper by Lin and Chen develops an efficient algorithm to search for the optimal assignment for the problem of double resource optimization for a robust computer network subject to a transmission budget problem.

The paper by Tamura and Yamada focuses on a cloud computing environment by proposing a new approach to software reliability assessment based on the neural network and jump diffusion models based on stochastic differential equations. The paper by Okamura and Dohi discusses a modeling framework of a software reliability model with phase-type distributions and develops parameter estimation algorithms with grouped data using the expectation-maximization approach. The paper by Inoue, Ikeda, and Yamada develops a bivariate software reliability growth model considering the uncertainty of the change of software failure-occurrence phenomenon and the effect at change-point by reflecting the difference of the software failure occurrence pattern before and after change-points.

The paper by Lin and Huang proposes an efficient algorithm to obtain the reliability of multi-state computer networks by determining the minimal capacity vector with considerations of both assured accuracy rate and time constraint. Finally, the paper by Fiondella and Zeephongsekul develops a trivariate Bernoulli distribution to quantify the influence of correlated failures on the reliability of fault tolerant software systems composed of highly reliable versions.

Special thanks are due to the Editor-in-Chief, Dr. Endre Boros, for his support and encouragement; to the reviewers for their valuable comments that helped to further improve the quality of the papers; and to the authors of all papers submitted to this issue. 